\title{
Isospin effects and the density dependence of the nuclear symmetry energy
}

\author{
S. R. Souza, ${ }^{1,2}$ M. B. Tsang, ${ }^{3}$ B. V. Carlson, ${ }^{4}$ R. Donangelo,,${ }^{1,5}$ W. G. Lynch,${ }^{3}$ and A. W. Steiner ${ }^{3}$ \\ ${ }^{1}$ Instituto de Física, Universidade Federal do Rio de Janeiro, Cidade Universitária, CP 68528, 21941-972, Rio de Janeiro, Brazil \\ ${ }^{2}$ Instituto de Física, Universidade Federal do Rio Grande do Sul, Av. Bento Gonçalves 9500, CP 15051, 91501-970, Porto Alegre, Brazil \\ ${ }^{3}$ Joint Institute for Nuclear Astrophysics, National Superconducting Cyclotron Laboratory, and the Department of Physics and Astronomy, \\ Michigan State University, East Lansing, Michigan 48824, USA \\ ${ }^{4}$ Departamento de Física, Instituto Tecnológico de Aeronáutica-CTA, 12228-900 São José dos Campos, Brazil \\ ${ }^{5}$ Instituto de Física, Facultad de Ingeniería, Universidad de la República, Julio Herrera y Reissig 565, 11.300 Montevideo, Uruguay
}

(Received 10 July 2009; published 22 October 2009)

\begin{abstract}
The density dependence of the nuclear symmetry energy is inspected using the statistical multifragmentation model with Skyrme effective interactions. The model consistently considers the expansion of the fragments' volumes at finite temperature at the freeze-out stage. By selecting parametrizations of the Skyrme force that lead to very different equations of state for the symmetry energy, we investigate the sensitivity of the isoscaling parameter and the isotopic distributions to differences in the symmetry energy. Our results suggest that, in spite of being sensitive to the thermal dilation of the fragments' volumes, it is difficult to distinguish among the Skyrme forces from the isoscaling analysis. On the other hand, the isotopic distribution of the emitted fragments turns out to be very sensitive to the force employed in the calculation.
\end{abstract}

DOI: 10.1103/PhysRevC.80.041602

PACS number(s): 25.70.Pq, 21.65.Ef, 24.60.-k, 21.30.Fe

Investigations of the density dependence of the symmetry energy in the nuclear multifragmentation process have been stimulated by the discovery $[1,2]$ that the ratio $R_{21}$ between the experimental yields $Y(A, Z)$ of a fragment of mass and atomic numbers $A$ and $Z$, respectively, produced in similar reactions with different isospin compositions, henceforth labeled 1 and 2 , follows a scaling law [3-16]. This isoscaling law provides parameters $\alpha$ and $\beta$ that are determined from the property

$$
R_{21} \equiv \frac{Y_{2}(A, Z)}{Y_{1}(A, Z)}=C \exp (\alpha N+\beta Z),
$$

where $C$ is a normalization constant [1].

Studies of the density dependence of the symmetry energy have used Eq. (1) to probe (i) the dependence of statistical models on the symmetry energy [7-9,13] and (ii) the isospin composition of the region emitting the fragments $[3,17,18]$. In this paper, we are concerned with the former issue. The motivation for statistical model studies arises from the relationship between the isoscaling parameter $\alpha$ and the nuclear symmetry energy, whose leading term at low temperatures was shown to be $[15,16]$

$$
\alpha \approx 4 C_{\mathrm{sym}}\left[\left(Z_{1} / A_{1}\right)^{2}-\left(Z_{2} / A_{2}\right)^{2}\right] / T,
$$

where $C_{\text {sym }}$ denotes the symmetry energy coefficient [6], $T$ is the temperature of the system, and $Z_{i}$ and $A_{i}(i=1,2)$ correspond, respectively, to the atomic and mass numbers of the decaying source.

To be useful, reactions 1 and 2 should be chosen to produce systems (sources) at approximately the same temperature and density. The ratio in Eq. (1) involves yields of the same isotope; distorting effects associated with the deexcitation of the primordial hot fragments may be similar in the two reactions and lead to an approximate cancellation in the ratio. Theoretical calculations support this assumption for primary distributions calculated from equilibrium statistical models [13-15]. Therefore, measurements of the isoscaling parameters, through fits based on Eq. (1), may provide valuable information on the symmetry energy.

This assumption has extensively been exploited in many works [7-13] which employed the statistical multifragmentation model (SMM) [19]. To reproduce the measured $\alpha$ parameter, $C_{\text {sym }}$ has been appreciably reduced compared with the values usually adopted in this model. The main conclusion of those works [7-13] is that there seems to be an important decrease of the symmetry energy at low densities.

Although this result is reasonable on physical grounds, other studies [4-6] have also provided a sound explanation for this apparent reduction of $C_{\text {sym }}$. They suggest that surface effects associated with the symmetry energy, not considered in Refs. [7-13], may also lead to significant reduction of the $\alpha$ parameter. This explanation seems to be more reasonable since the model used in all these studies [4-13] is based on binding energy formulas that evaluate the isoscalar volume, surface, and Coulomb terms at the ground state (saturation) density. Although the volume occupied by the total system is much larger than that of the ground state source $V_{0}$, the lower density values used in these statistical calculations are due to the space between the fragments. Therefore, in this scenario, there should be no sensitivity to the density dependence of the bulk symmetry energy.

In this work, we investigate this issue in a consistent approach in which the properties of the fragments are calculated through the Thomas-Fermi approximation (TFA) at finite temperature. More specifically, for each fragment, the equilibrium density and different contributions to the Helmholtz free energy at temperature $T$ are determined by a microscopic TFA mean-field calculation. The SMM then employs these equilibrium properties of the fragments at temperature $T$ to determine the statistical weight of the different fragmentation modes. This version of the SMM, named SMM-TF, has been presented in detail in Ref. [20] and is briefly sketched below. 
In the microcanonical models employed in this work $[20,21]$, the statistical importance of a fragmentation mode $f$, which fulfills mass, charge, and energy constraints, is given by the number of microstates $\mathcal{N}_{f}=\exp \left(S_{f}\right)$ accessible to the fragmentation mode at a given energy. The entropy $S_{f}$ is calculated through the Helmholtz free energy $F(T)$ associated with the fragmentation mode $f$. In both the SMM-TF [20] and the improved statistical multifragmentation model (ISMM) [21], also used in this work, $F$ is written as

$$
\begin{aligned}
F(T)= & \frac{C_{\text {Coul }}}{(1+\chi)^{1 / 3}} \frac{Z_{0}^{2}}{A_{0}^{1 / 3}}+F_{\text {trans }}(T) \\
& +\sum_{A, Z} N_{A, Z}\left[-B_{A, Z}+f_{A, Z}^{*}(T)-\frac{C_{\text {Coul }}}{(1+\chi)^{1 / 3}} \frac{Z^{2}}{A^{1 / 3}}\right],
\end{aligned}
$$

where

$$
\begin{aligned}
F_{\text {trans }}= & -T(M-1) \log \left(V_{f} / \lambda_{T}^{3}\right)+T \log \left(g_{0} A_{0}^{3 / 2}\right) \\
& -T \sum_{A, Z} N_{A, Z}\left[\log \left(g_{A, Z} A^{3 / 2}\right)-\frac{1}{N_{A, Z}} \log \left(N_{A, Z} !\right)\right]
\end{aligned}
$$

In the above equations, $C_{\text {Coul }}$ denotes the Coulomb coefficient of the mass formula [21], $A_{0}$ and $Z_{0}$ are the mass and atomic numbers of the decaying source, $B_{A, Z}$ represents the binding energy of the fragment, $N_{A, Z}$ stands for its multiplicity, and $M=\sum_{A, Z} N_{A, Z}$. The freeze-out volume $V_{\chi}=V_{0}(1+\chi)$ is kept fixed $(\chi=2)$ for all the values of the source's excitation energy $E^{*}$. The factor $M-1$, rather than $M$, as well as $T \log \left(g_{0} A_{0}^{3 / 2}\right)$, in the translational contribution to the free energy, arise from the subtraction of the center of mass motion from the partition function of the total system. The spin degeneracy factor is denoted by $g_{A, Z}, \lambda_{T}=\sqrt{2 \pi \hbar^{2} / m_{n} T}$ corresponds to the thermal wavelength, and $m_{n}$ is the nucleon mass. As in the ISMM, the free volume reads

$$
V_{f}(T)=V_{0}(1+\chi)-\sum_{A, Z} N_{A, Z} V_{A, Z}(T),
$$

where $V_{A, Z}(T)$ is the volume occupied by each fragment. However, in the SMM-TF, $V_{A, Z}(T)$ is given by the TFA [20], and therefore it differs from the ground state value used in the ISMM. The internal Helmholtz free energy of the fragment $f_{A, Z}^{*}$ in the SMM-TF model is also given by this microscopic approach. These are the only two differences between the SMM-TF and the ISMM, as discussed in Ref. [20]. All the other ingredients are the same.

Owing to the plethora of Skyrme forces in the literature [22,23], we do not perform a complete study using all the existing parametrizations. Instead, we selected two of them that give distinct equations of state for the symmetry energy, i.e., the Gs [24] and the SLy4 [25] forces. Figure 1 shows the density dependence of the symmetry energy coefficient of cold nuclear matter, $\delta E_{\mathrm{sym}} / A=C_{\mathrm{sym}} \delta^{2}$, where $\delta=\left(\rho_{n}-\right.$ $\left.\rho_{p}\right) /\left(\rho_{n}+\rho_{p}\right)$ and $\rho_{n}\left(\rho_{p}\right)$ is the neutron (proton) density. One sees that although both forces agree for densities close to the saturation value $\left(\rho_{0}\right)$, the differences at lower densities can be appreciably large. The Gs and SLy4 forces provide

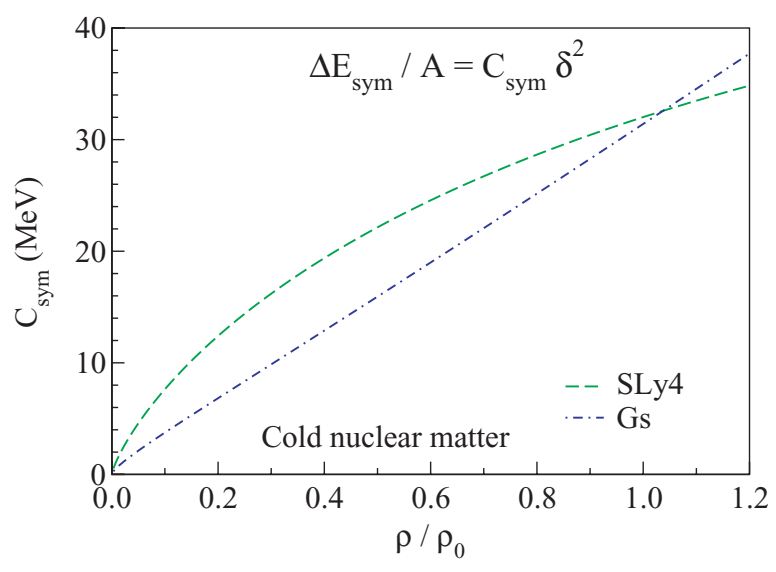

FIG. 1. (Color online) Symmetry energy coefficient of cold nuclear matter for the SLy4 and Gs Skyrme forces as a function of the density $\rho$.

examples of a strongly density-dependent (stiff) and a weakly density-dependent (soft) symmetry energy, respectively. Both have bulk isoscalar incompressibility moduli in the range of 230 to $250 \mathrm{MeV}$ [26]. It should be noted that most of the Skyrme forces satisfying this property, in addition to giving similar values at the saturation density, yield symmetry energy curves that lie within those obtained with the Gs and SLy4 forces. Therefore, our particular choice is well suited for the present study.

We confine our attention to the ${ }^{40} \mathrm{Ar},{ }^{40} \mathrm{Ca}+{ }^{58} \mathrm{Ni}$ systems, for which measurements of the isoscaling parameter $\alpha$ have been reported recently [13]. The decaying sources considered in the calculations below correspond to $80 \%$ of the compound systems, as $20 \%$ of the matter is removed in order to take the preequilibrium emission into account. To make a connection to the work of Ref. [13], we adopt in the following their assumption that the $Z / A$ of the source is the same as the original system.

We begin our discussion by examining whether the isoscaling property should still hold if the thermal expansion of the fragments' volumes is taken into account. The microcanonical treatment employed in the SMM-TF does not allow one to derive analytical expressions to investigate this issue. Furthermore, the dependence of the free volume on the species multiplicities, Eq. (5), leads to highly nonlinear terms in the Helmholtz free energy, rendering the traditional grandcanonical formulas [6] invalid. By minimizing $F$ with respect to the multiplicities, as done in Ref. [6], one may nevertheless obtain expressions that allow one to address this issue. More specifically, this minimization procedure gives

$$
\begin{aligned}
f_{k}- & T\left[\log \left(\frac{g_{k}}{\lambda_{T}^{3}} V_{f} A_{k}^{3 / 2}\right)-\log \left(n_{k}\right)+1\right]+T \\
& +\frac{T}{V_{f}(T)} V_{k}(T) \sum_{i} n_{i}-\mu_{B} A_{k}-\mu_{Q} Z_{k}=0,
\end{aligned}
$$

where $f_{k}$ corresponds to the term within the brackets in Eq. (3), $n_{k}$ is the average multiplicity of species $k$, and $\mu_{B}$ and $\mu_{Q}$ stand for the baryon and charge chemical potentials. By denoting the average total multiplicity as $\langle M\rangle=\sum_{k} n_{k}$, 
the above expression can be formally written as

$$
n_{k}=\frac{g_{k} V_{f} A_{k}^{3 / 2}}{\lambda_{T}^{3}} e^{-\left[f_{k}-\mu_{B} A_{k}-\mu_{Q} Z_{k}+\frac{V_{k}}{V_{f}} T\langle M\rangle\right] / T} .
$$

Note that although this equation is similar to the traditional formula [6], it is not a closed expression, since $V_{f}$ depends strongly on the fragments' multiplicities. Therefore, the behavior of the chemical potentials should be expected to be very different from the case in which the fragments' volumes do not expand. In the latter scenario, on which the ISMM is based, $V_{f}$ does not depend on the fragmentation mode, so $\frac{V_{k}}{V_{f}} T\langle M\rangle=\left[\frac{1}{\chi} T\langle M\rangle / A_{0}\right] A_{k}$. In this case, since the term within the brackets does not depend on $A_{k}$ or $Z_{k}$, this factor may be absorbed by $\mu_{B}$ and the traditional formula [6] is recovered. However, this is not the case in the SMM-TF model.

Nevertheless, by inserting Eq. (7) into Eq. (1), one obtains

$$
R_{21}=e^{\left[\frac{\Delta \mu_{B}}{T} A_{k}+\frac{\Delta \mu_{Q}}{T} Z_{k}+\frac{V_{k}}{V_{f}}\left(\left\langle M_{1}\right\rangle-\left\langle M_{2}\right\rangle\right)\right]},
$$

where $\Delta \mu=\mu^{(2)}-\mu^{(1)}$ is the difference between the chemical potentials associated with the sources in the two reactions. The dependence of the last term of the exponential on $n_{k}$ may lead to important distortions that could invalidate the isoscaling law in the framework of the SMM-TF. However, this term is expected to be small since $\left\langle M_{1}\right\rangle \approx\left\langle M_{2}\right\rangle$, as the decaying sources should be similar, and also because the free volume $V_{f}$ is usually much larger than the fragment volume $V_{k}$ (except for $T$ close to the limiting temperature). Thus, one should expect the isoscaling property to remain valid.

This is indeed found in our numerical microcanonical SMM-TF calculations, as illustrated in Fig. 2, which shows $R_{21}$ for the SLy4 force and $E^{*} / A=6.0 \mathrm{MeV}$. Similar results are obtained for the $G$ s force and for other excitation energies. The magnitude of the corrections due to the deexcitation of the hot primary fragments can be estimated by comparing the upper and lower panels of Fig. 2. Here, we simulated the decay with a simplified Monte Carlo Weisskopf model, which includes the emission of nuclei up to oxygen. The parametrization of the cross section for the inverse reaction was taken from Ref. [27].

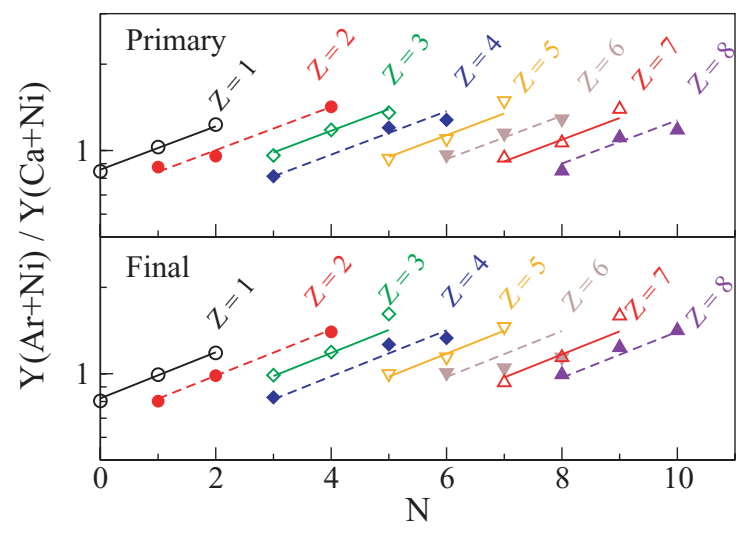

FIG. 2. (Color online) Isoscaling predicted by the SMM-TF using the SLy4 for $E^{*} / A=6.0 \mathrm{MeV}$. In the upper panel, the yields of the hot primary fragments are used; the lower panel displays the results after the deexcitation of these fragments. The lines correspond to the best fit of the results using Eq. (1).

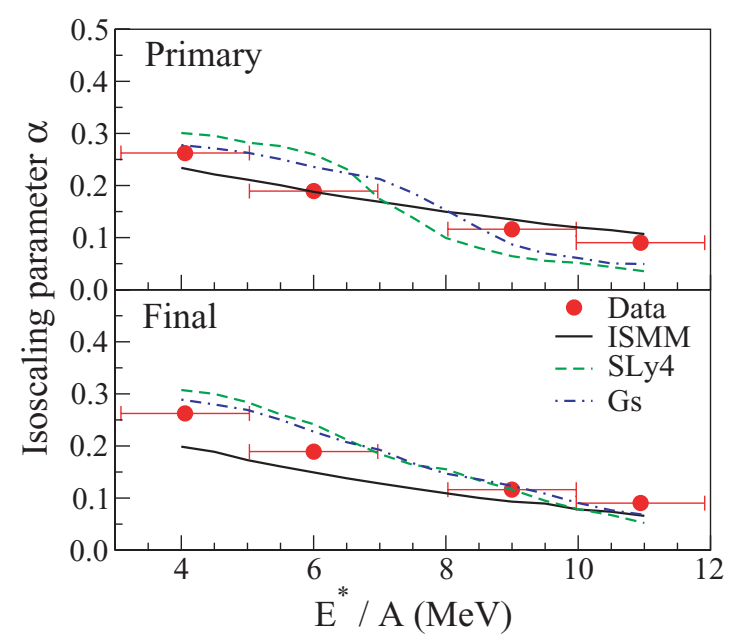

FIG. 3. (Color online) Isoscaling parameter $\alpha$ as a function of the excitation energy of the source, for different Skyrme forces and the ISMM, before and after the deexcitation of the primary fragments. The data are from Ref. [13].

Following Ref. [28], we calculate the density of states from the entropies associated with $f_{A, Z}^{*}$. This provides a consistent link between the primary stage and the deexcitation process. In agreement with previous calculations [13-15], our results also suggest that $\alpha$ is not strongly sensitive to the deexcitation of the primary fragments.

Figure 3 shows the comparison between the $\alpha$ values obtained in the different SMM models used in this work and the available experimental data [13]. The results corresponding to the primary fragments (top panel of Fig. 3) reveal that $\alpha$ is fairly sensitive to the thermal dilatation of the fragments' volumes, as the behavior of $\alpha$ in the SMM-TF calculations is different from that given by the ISMM. Switching from the SLy4 to the Gs force leads to small differences in $\alpha$, primarily within the small energy range $7.0<E^{*} / A<8.5 \mathrm{MeV}$. This corresponds to the region where the average fragment density is approximately $0.65 \rho_{0}$. For the ISMM, investigations of the connection between $\alpha$ and $C_{\text {sym }}$ at finite temperatures show that Eq. (2) can be fairly inaccurate at high temperatures, although the main conclusion that both quantities are strongly correlated remains valid [29].

It is interesting to examine whether comparisons of the SMM-TF calculations to experimental fragmentation data can clarify questions regarding the adequacy of the model and the effective forces selected to describe the multifragment emission. The ISMM results seem to follow the experimental trends more closely than the SMM-TF calculations. This picture does not survive the deexcitation of the primary fragments. Although the changes to $\alpha$ are small, the values predicted by the ISMM are systematically lowered so that, after secondary decay, they lie below the data and the SMM-TF results. The differences between the calculations with the SLy4 and the Gs forces are very small after the decay of the primary fragments, and our results suggest that it is difficult to distinguish between them from the isoscaling analysis.

Distinct Skyrme forces lead to different values of $V_{A, Z}(T)$ [30]. The Helmholtz free energy is sensitive to the free 


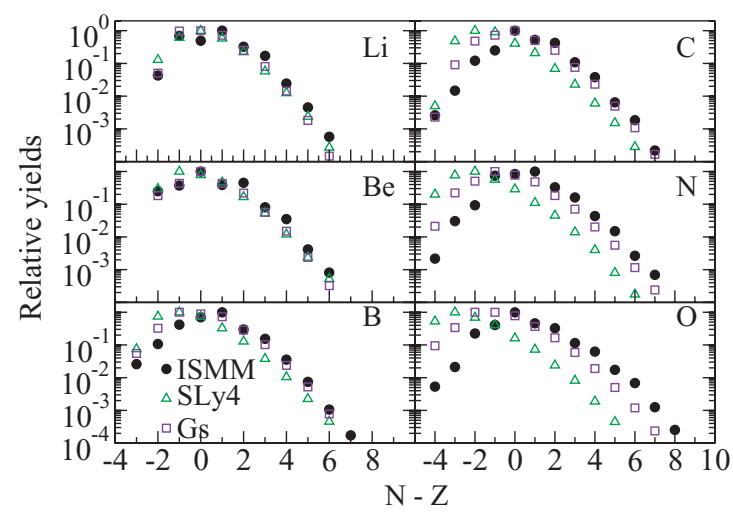

FIG. 4. (Color online) Isotopic distribution of selected primary fragments for the $\mathrm{Ar}+\mathrm{Ni}$ system at $E^{*} / A=8.5 \mathrm{MeV}$.

volume, which is directly affected by the thermal expansion of the fragments [Eqs. (4) and (5)]. Therefore, the same fragmentation mode will give different contributions to $F(T)$ for different Skyrme forces. As a consequence, the relative statistical weight of this fragmentation mode depends on the Skyrme force employed, which leads to distinct isotopic distributions. This is indeed observed in Fig. 4, which displays the isotopic distribution of selected primary hot nuclei for the breakup of the $\mathrm{Ar}+\mathrm{Ni}$ system at $E^{*} / A=8.5 \mathrm{MeV}$. It has been shown in Ref. [20] that, at low energies, the isotopic distributions predicted by the SMM-TF are narrower than those given by the ISMM. However, they are very different at higher excitation energies, as shown in Fig. 4, where, for this system, the peak of the distribution shifts toward the proton-rich isotopes. The effect is enhanced for higher excitation energies, and it is more pronounced in the case of the SLy4 force than for the Gs force. We have checked that, although these trends remain true, the differences between the Gs and the SLy4 forces are reduced after the decay of the primary fragments, whereas they remain large enough to clearly distinguish between the ISMM and the SMM-TF calculations. To draw precise conclusions, a deexcitation treatment that explicitly takes the feeding between known discrete states of these fragments into account should be developed and applied to the SMM-TF, as it has been demonstrated in Ref. [21] that the isotopic distribution is fairly sensitive to this ingredient.
When such a treatment has been implemented, one will be able to reliably investigate whether the uncertainties associated with the deexcitation of the primary fragments will allow one to distinguish among different SMM calculations through confrontation with the experimental isotopic distributions.

In summary, we have tested, self-consistently, the sensitivity of equilibrium multifragmentation theories to the density dependence of the symmetry energy. Our statistical calculations, which consistently incorporate Skyrme effective interactions, suggest that the corresponding predicted differences in the isoscaling parameter $\alpha$ are not large enough to allow one to distinguish between symmetry energies with very different density dependencies. This observation does not impact the use of $\alpha$ to probe the asymmetry of the emitting system, a use that has been instrumental in investigations of the density-dependent symmetry energy $[3,17,18]$. On the other hand, the isotopic distribution of the fragments produced in the reactions retains some sensitivity to the effective force employed in the calculations. We believe that constraining the key SMM-TF inputs, i.e., the source composition and the excitation energy, through experimental values of additional observables besides the isoscaling parameters, will be critical to precision comparisons aimed at constraining the symmetry energy with isotopic distributions. The isotopic composition of the decaying source is a quantity that is very difficult to constrain by direct measurements, as information on the freeze-out configuration of the system is usually biased by the deexcitation of the hot primordial fragments. The restrictions imposed by the comparison with other observables may lead to input sources to the model whose $Z / A$ ratios are different for distinct Skyrme forces. Since the isoscaling is very sensitive to the $Z / A$ ratio of the sources [15], the small differences found in the present work may be enhanced if different $Z / A$ values are required for different Skyrme forces. However, such detailed study requires the elaboration of a deexcitation scheme, such as that described in Ref. [21].

We would like to acknowledge CNPq, FAPERJ, CNPqPROSUL, and the PRONEX program under Contract No. E-26/171.528/2006, for partial financial support. This work was supported in part by the National Science Foundation under Grant Nos. PHY-0606007 and INT-0228058. A.W.S. is supported by NSF Grant No. 04-56903.
[1] H. S. Xu et al., Phys. Rev. Lett. 85, 716 (2000).

[2] M. B. Tsang, W. A. Friedman, C. K. Gelbke, W. G. Lynch, G. Verde, and H. S. Xu, Phys. Rev. Lett. 86, 5023 (2001).

[3] A. Ono, P. Danielewicz, W. A. Friedman, W. G. Lynch, and M. B. Tsang, Phys. Rev. C 68, 051601(R) (2003).

[4] Ad. R. Raduta and F. Gulminelli, Phys. Rev. C 75, 024605 (2007).

[5] Ad. R. Raduta and F. Gulminelli, Phys. Rev. C 75, 044605 (2007).

[6] S. R. Souza, M. B. Tsang, R. Donangelo, W. G. Lynch, and A. W. Steiner, Phys. Rev. C 78, 014605 (2008).

[7] A. Le Fèvre et al., Phys. Rev. Lett. 94, 162701 (2005).

[8] D. V. Shetty, S. J. Yennello, and G. A. Souliotis, Phys. Rev. C 76, 024606 (2007).
[9] A. S. Botvina, N. Buyukcizmeci, M. Erdogan, J. Łukasik, I. N. Mishustin, R. Ogul, and W. Trautmann, Phys. Rev. C 74, 044609 (2006).

[10] S. Kowalski et al., Phys. Rev. C 75, 014601 (2007).

[11] G. A. Souliotis, A. S. Botvina, D. V. Shetty, A. L. Keksis, M. Jandel, M. Veselsky, and S. J. Yennello, Phys. Rev. C 75, 011601(R) (2007).

[12] N. Buyukcizmeci, R. Ogul, and A. S. Botvina, Eur. Phys. J. A 25, 57 (2005).

[13] J. Iglio et al., Phys. Rev. C 74, 024605 (2006).

[14] S. R. Souza, R. Donangelo, W. G. Lynch, W. P. Tan, and M. B. Tsang, Phys. Rev. C 69, 031607(R) (2004).

[15] M. B. Tsang et al., Phys. Rev. C 64, 054615 (2001).

[16] A. S. Botvina, O. V. Lozhkin, and W. Trautmann, Phys. Rev. C 65, 044610 (2002). 
[17] M. B. Tsang et al., Phys. Rev. Lett. 92, 062701 (2004).

[18] W. P. Tan et al., Phys. Rev. C 64, 051901(R) (2001).

[19] J. P. Bondorf, A. S. Botvina, A. S. Iljinov, I. N. Mihustin, and K. Sneppen, Phys. Rep. 257, 133 (1995)

[20] S. R. Souza, B. V. Carlson, R. Donangelo, W. G. Lynch, A. W. Steiner, and M. B. Tsang, Phys. Rev. C 79, 054602 (2009).

[21] W. P. Tan, S. R. Souza, R. J. Charity, R. Donangelo, W. G. Lynch, and M. B. Tsang, Phys. Rev. C 68, 034609 (2003).

[22] J. R. Stone, J. C. Miller, R. Koncewicz, P. D. Stevenson, and M. R. Strayer, Phys. Rev. C 68, 034324 (2003).

[23] P. Danielewicz and J. Lee, Nucl. Phys. A818, 36 (2009).
[24] J. Friedrich and P.-G. Reinhard, Phys. Rev. C 33, 335 (1986).

[25] F. Douchin and P. Haensel, Phys. Lett. B485, 107 (2000).

[26] G. Colò, N. V. Giai, J. Meyer, K. Bennaceur, and P. Bonche, Phys. Rev. C 70, 024307 (2004).

[27] A. S. Iljinov, I. N. Mishustin, J. P. Bondorf, R. Donangelo, and K. Sneppen, Nucl. Phys. A475, 663 (1987).

[28] H. W. Barz, J. P. Bondorf, R. Donangelo, I. N. Mishustin, and H. Schulz, Nucl. Phys. A448, 753 (1986).

[29] S. R. Souza, M. B. Tsang, B. V. Carlson, R. Donangelo, W. G. Lynch, and A. W. Steiner, Phys. Rev. C 80, 044606 (2009).

[30] P. Bonche, S. Levit, and D. Vautherin, Nucl. Phys. A436, 265 (1985). 